\title{
DIETA DE FINES DE VERANO DEL HUEMUL, HIPPOCAMELUS BISULCUS, EN EL VALLE DEL RÍO DE LAS VUELTAS, ARGENTINA
}

\author{
SUMMER DIET OF HUEMUL, HIPPOCAMELUS BISULCUS, \\ IN RÍO DE LAS VUELTAS VALLEY, ARGENTINA
}

Alejandro Ruben Vila ${ }^{1}$, Laura Borrelli ${ }^{2}$ \& Gustavo Aprile ${ }^{3}$

El huemul (Hippocamelus bisulcus Molina, 1782) es un ciervo de tamaño mediano endémico de Chile y Argentina. Originalmente se distribuía en la región andina patagónica y sus áreas ecotonales con la estepa (Cabrera \& Yepes 1960), entre los $34^{\circ} \mathrm{S}$ y $54^{\circ} \mathrm{S}$ (Díaz 2000). Sin embargo, tanto su distribución como sus poblaciones se redujeron significativamente (Drouilly 1983, Redford \& Eisenberg 1992, Serret 1992). Por esta razón, el huemul ha sido clasificado como "amenazado" en ambos países (Glade 1988, Díaz \& Ojeda 2000) y a nivel internacional (Jiménez et al. 2008).

A pesar de que los estudios sobre la biología y ecología del huemul son escasos (Díaz \& SmithFlueck 2000, Serret 2001), la composición de su dieta y/o selección de alimento ha sido descrita en algunos sitios de Chile (Colomes 1978¹, Frid 1994, López et al. 2001, Van Winden 2006²) y Argentina (Merino 1995, Smith-Flueck 2003³, Galende et al. 2005, Vila et al. 2009a, 2009b). Estos estudios han demostrado que el huemul exhibe un patrón dietario caracterizado por el consumo de especies leñosas y herbáceas (Vila et al. 2009a, 2010), lo cual es consistente con los hábitos alimentarios de un selector concentrado (Hofmann 1989).

La distribución actual de la especie se extiende a lo largo de una estrecha franja de $1900 \mathrm{~km}$, entre

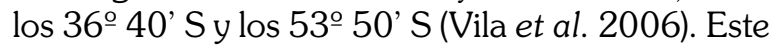
amplio rango latitudinal incluye condiciones altamente variables de altitud (desde el nivel del mar a los $3.000 \mathrm{msnm}$ ), precipitaciones (800 a $5.000 \mathrm{~mm}$ ), tipos de vegetación y hábitats (Vila et al. 2010). Esta

1 Wildlife Conservation Society, Balmaceda 586, Punta Arenas, Chile. alevila@speedy.com.ar.

2 Instituto Nacional de Tecnología Agropecuaria (INTA), EEA-Bariloche, Modesta Victoria 4450, (8400) Bariloche, Río Negro, Argentina.

3 S.A.S./ACEN, Serrano 1779 (1663) San Miguel, Buenos Aires, Argentina.

1 Colomes, A. 1978. Biología y ecología del huemul chileno (Hippocamelus bisulcus): Estudios de sus hábitos alimentarios. Tesis Doctoral, Universidad de Chile. Santiago, Chile.

2 Van Winden, J. 2006. Diet and habitat of the huemul (Hippocamelus bisulcus) in Bernardo O'Higgins National Park, Chile. Master's thesis, Utrecht University. Holanda.

3 Smith-Flueck, J.A. 2003. La ecología del huemul (Hippocamelus bisulcus) en la Patagonia Andina de Argentina y consideraciones sobre su conservación. Tesis Doctoral, Universidad Nacional del Comahue. Bariloche, Argentina. 
diversidad de condiciones ambientales, junto con las amenazas que enfrenta cada población, podrían influenciar los patrones de alimentación del huemul dentro de su rango de distribución.

En la porción austral de su distribución se ha evaluado su ecología alimentaria, por medio de la observación directa de ejemplares o mediante la técnica de microhistología, en tres poblaciones que se encuentran aisladas entre sí, Torres del Paine (Guineo et al. 20084), Fiordo Témpanos (Frid 1994, Van Winden 2006) y Seno Moyano (Merino 1995). En este trabajo reportamos la composición de la dieta de fines del verano para una población austral de huemules ubicada en la Provincia de Santa Cruz, Argentina.

El estudio se realizó en el margen occidental del valle del río de Las Vueltas e incluyó la porción norte del Parque Nacional Los Glaciares y la estancia. Los Huemules, una reserva privada ubicada unos $20 \mathrm{~km}$ al norte de la localidad de El Chaltén. La vegetación del área de estudio se corresponde con las Provincias Fitogeográficas Subantártica, Distrito de los Bosques Caducifolios, y Altoandina (Cabrera 1976). Los bosques están dominados por lenga (Nothofagus pumilio) y ñire (Nothofagus antarctica), mientras que las praderas y matorrales altoandinos se desarrollan a partir de los $800 \mathrm{msnm}$. Las condiciones climáticas invernales son rigurosas, con nevadas y heladas, y contrastan con las altas temperaturas y larga duración de los días en verano (Quintana et al. 2003). El huemul presenta una amplia distribución en este sitio, pero se desconoce su tamaño poblacional (López et al. 1998, Vila et al. 2006). Los pobladores de la región se dedicaban a la cría extensiva de ganado, pero en la actualidad está ocurriendo un proceso de conversión parcial o total al uso turístico de la tierra. Por esta razón, las áreas prospectadas han estado libres de ganado, al menos, durante los últimos 10 años.

Como parte de un relevamiento expeditivo para determinar la presencia de huemules, se realizaron transectos perpendiculares a las curvas de nivel cubriendo desde el valle hasta el prado de altura. Se colectaron un total de siete fecas (bostas) frescas entre los 440 y $670 \mathrm{msnm}$, entre el 17 y

4 Guineo, O., R. Guineo Garay \& G. Garay 2008. Conociendo al huemul de Torres del Paine. La Prensa Austral, Punta Arenas, Chile. el 20 de marzo de 2007, en los siguientes sitios: Faldeo del Cerro Polo (49 ${ }^{\circ} 15^{\prime} 7,53$ 'S - 72 $56^{\circ}$ 35,83"O), el Chorrillo del Salto (49 $17^{\circ}$ '39,97"S - 72 54' 33,83"O) y la Loma del Diablo (49 $11^{\circ}$ $42,16 " S$ - 72 57' 43,10"O).

La composición botánica de la dieta fue obtenida a través del análisis microhistológico de heces, por identificación de fragmentos epidérmicos (Sparks \& Malechek 1968) y no epidérmicos de las plantas consumidas (Sepúlveda et al. 2004). Las muestras se secaron en estufa a $60{ }^{\circ} \mathrm{C}$ durante 48 horas, se molieron con un molino tipo Willey (malla de 1 $\mathrm{mm}$ ), despigmentaron con alcohol 70\%, aclararon con hipoclorito de sodio y montaron en gelatina glicerina (Sparks \& Malechek 1968, Williams 1969, Latour \& Pelliza Sbriller 1981). Se montaron cinco preparados por muestra y se observaron 20 campos microscópicos a 100 aumentos por cada preparado (Holechek \& Vavra 1981), totalizando 700 campos de observación. Los fragmentos observados en las muestras se identificaron a nivel de género, y cuando fue posible a nivel de especie, a partir del estudio de tejidos de una colección de plantas de referencia previamente colectada en la región. La clasificación botánica de las plantas siguió la nomenclatura propuesta por el Instituto de Botánica Darwinion (www.darwin.edu.ar). La cuantificación de los ítems alimentarios determinados en la dieta fue realizada a partir de frecuencias relativas (Holechek \& Gross 1982). Las especies fueron agrupadas en las siguientes formas de vida: árboles, arbustos, hierbas y gramíneas (incluyendo graminoideas). La amplitud de nicho trófico fue calculada por medio del índice normalizado de Levins (Krebs 1989).

La dieta estival del huemul en el valle del río de Las Vueltas estuvo compuesta por 26 especies vegetales (Tabla 1). Las formas de vida más representadas dentro de la dieta fueron las herbáceas (10 especies) y las gramíneas (8 especies). El huemul consumió principalmente plantas leñosas, 30,6 y 36,8\% de los fragmentos de árboles y arbustos registrados en las heces, respectivamente. El aporte de hierbas en su dieta también fue importante (23,8\%), mientras que el consumo de gramíneas sólo alcanzó el 8,8\%. Sólo cuatro especies aportaron el 68\% de los fragmentos de plantas registrados en las muestras, Nothofagus sp., los arbustos Adesmia volckmannii y Maytenus sp., y la herbácea Gunnera magellanica (Tabla 1). La dieta del huemul estuvo compuesta por menos 
Tabla 1: Composición de la dieta del huemul en el valle del río de Las Vueltas hacia fines del verano.

\begin{tabular}{|c|c|c|}
\hline ESPECIES & MEDIA & ERROR ESTÁNDAR \\
\hline \multicolumn{3}{|l|}{ ÁRBOLES } \\
\hline Lepidothamnus fonckii & 0,94 & 0,94 \\
\hline Nothofagus sp. & 29,61 & 5,18 \\
\hline \multicolumn{3}{|l|}{ ARBUSTOS } \\
\hline Adesmia volckmannii & 10,73 & 2,61 \\
\hline Discaria sp. & 4,65 & 1,79 \\
\hline Fuchsia magellanica & 0,05 & 0,05 \\
\hline Gaultheria mucronata & 4,31 & 1,44 \\
\hline Maytenus sp. & 17,05 & 5,74 \\
\hline Mulinum spp. & 0,05 & 0,05 \\
\hline \multicolumn{3}{|l|}{ HIERBAS } \\
\hline Cerastium fontanum & 4,13 & 3,94 \\
\hline Erodium spp. & 1,18 & 0,84 \\
\hline Fruto sin identificar & 1,25 & 1,11 \\
\hline Gunnera magellanica & 10,55 & 2,76 \\
\hline Hierba sin identificar & 0,52 & 0,38 \\
\hline Luzuriaga marginata & 0,57 & 0,39 \\
\hline Medicago lupulina & 1,64 & 1,51 \\
\hline Misodendron sp. & 1,00 & 0,82 \\
\hline Philesia magellanica & 2,02 & 1,66 \\
\hline Rubus sp. & 0,94 & 0,49 \\
\hline \multicolumn{3}{|l|}{ GRAMÍNEAS } \\
\hline Alopecurus sp. & 0,38 & 0,38 \\
\hline Bromus sp. & 2,91 & 2,42 \\
\hline Carex sp. & 0,25 & 0,18 \\
\hline Gramínea sin identificar & 0,48 & 0,25 \\
\hline Holcus sp. & 0,09 & 0,09 \\
\hline Poa sp. & 4,15 & 4,15 \\
\hline Rytidosperma sp. & 0,09 & 0,09 \\
\hline Stipa sp. & 0,47 & 0,35 \\
\hline
\end{tabular}

del 5\% de las restantes especies identificadas en los preparados, siendo Poa $s p$. la gramínea más importante (Tabla 1). El índice de amplitud de nicho fue bajo $(0,23)$.

El número de especies registrado en la dieta estival del huemul en el valle del río de Las Vueltas fue semejante al documentado en otros estudios (Vila et al. 2009a, 2010). Sin embargo, Guineo et al. (2008) registraron el consumo de hasta 120 especies vegetales en el Parque Nacional Torres del Paine. A pesar de que estos autores no cuantificaron la composición de la dieta, mencionan que el huemul concentró su consumo en al menos cinco especies características del matorral, Embothrium coccineum, Maytenus magellanica, Escallonia rubra, Chiliotrichium diffusum y Senecio spp. Nuestro estudio también evidenció una dieta altamente concentrada en cuatro especies, tal como ha sido documentado en otros estudios (Vila et al. 2009a). Las especies más consumidas en el valle del río de Las Vueltas han sido reportadas como clave para la dieta del huemul a lo largo de su rango de distribución (Vila et al. 2009a; 2010). Asimismo, el valor de amplitud de nicho estival observado fue bajo y es consistente con una dieta especializada y concentrada en pocas especies. Valores similares de amplitud de nicho trófico han sido reportados para otras poblaciones andinas durante el verano (Galende et al. 2005, Vila et al. 2009a).

El estudio sobre hábitos alimentarios en una población ubicada a una latitud similar ha sido realizado en el Fiordo Témpanos (48 41'33"S - 73을 59'21"W), Parque Nacional Bernardo O'Higgins. En este sitio periglaciar, que se encuentra separado 
de nuestra área estudio por el Campo de Hielo Sur, tanto Frid (1994) como Van Winden (2006) evaluaron el tiempo de forrajeo de huemules identificados por marcas naturales. Según Frid (1994) la dieta estuvo mayormente compuesta por herbáceas, principalmente G. magellanica, durante la primavera. En cambio, Van Winden (2006) observó un consumo concentrado en especies herbáceas y leñosas entre el verano y el otoño, mayormente G. magellanica y Fucsia magellanica. La tercera población estudiada en la zona austral se encuentra en el Seno Moyano (493' 56,92"S - 72 54' 50,37”O) y está geográficamente aislada de nuestro sitio de estudio por el Campo de Hielo y el Lago Viedma. Merino (1995) describió allí una dieta compuesta por herbáceas (50\%), con un importante aporte de leñosas (38\%) y menos relevante de gramíneas (12\%). El porcentaje de herbáceas en nuestra área de estudio fue menor que al registrado en estas poblaciones vecinas, pero comparable al observado por Sierralta (2001) en el Lago Cochrane y Smith-Flueck (2003) en el Lago La Plata, ubicados a los $47^{\circ} \mathrm{S}$ y $44^{\circ} \mathrm{S}$ respectivamente, donde los porcentajes de hierbas en la dieta fueron 27 y 23\%. En cambio, la dieta de poblaciones de huemul estudiadas entre los $41^{\circ} \mathrm{S}$ y $42^{\circ} \mathrm{S}$ estuvo principalmente compuesta por plantas leñosas (Galende et al. 2005, Vila et al. 2009a). Los estudios realizados por Colomes (1978) y López et al. (2001) en la población más septentrional conocida, en los Nevados del Chillán (36은 ), mostraron un alto consumo de herbáceas (43 al 64\%) y leñosas (34\%). Finalmente, es importante destacar que el consumo reportado de gramíneas ha sido muy bajo en la mayoría de las poblaciones estudiadas, a excepción de nuestros resultados y los obtenidos para el Lago Cochrane (Sierralta 2001) y el Seno Moyano (Merino 1995).

\section{AGRADECIMIENTOS}

Este trabajo fue realizado gracias a las autorizaciones otorgadas por la Administración de Parques Nacionales y la Dirección de Fauna Silvestre y Áreas Protegidas del Consejo Agrario Provincial de Santa Cruz. El apoyo logístico brindado por el personal de la Ea. Los Huemules, la Delegación del Consejo Agrario Provincial de El Chaltén y el personal de la Seccional Lago Viedma del Parque Nacional Los Glaciares resultó crítico para concretarlo. En particular, agradecemos la colaboración de Norma Álvarez, Pablo Díaz, Carlos Duprés, Paz Fiorito, Susana Queiro, Federico Reese, Diego Ríos, Juan Manuel Sánchez, Alejandro Serret, Víctor Sotelo y Carlos Zoratti. También agradecemos los comentarios de dos revisores anónimos para mejorar la versión original del manuscrito.

\section{LITERATURA CITADA}

Cabrera, A. \& J. Yepes 1960. Mamíferos Sudamericanos. Segunda Edición. Ediar, Buenos Aires, Argentina.

Díaz, N.I. 2000. El huemul (Hippocamelus bisulcus Molina, 1782): Una perspectiva histórica. En: Díaz, N.I. \& J.A. Smith-Flueck (eds) El huemul Patagónico, un misterioso cérvido al borde de la extinción. pp: 1-32, Literature of Latin America, Buenos Aires, Argentina.

Díaz, N.I. \& J.A. Smith-Flueck 2000. El huemul Patagónico, un misterioso cérvido al borde de la extinción. Literature of Latin America, Buenos Aires, Argentina.

Díaz, G.B. \& R.A. Ojeda 2000. Libro Rojo. Mamíferos amenazados de la Argentina. SAREM, Mendoza, Argentina.

Drouilly, P. 1983. Recopilación de antecedentes biológicos y ecológicos del huemul chileno y consideraciones sobre su manejo. Boletín Técnico CONAF 5:1-57.

Frid, A. 1994. Observations on habitat use and social organization of a huemul (Hippocamelus bisulcus) coastal population in Chile. Biological Conservation 67:13-19.

Galende, G., E. Ramilo \& A. Beati 2005. Diet of Huemul Deer (Hippocamelus bisulcus) in Nahuel Huapi National Park, Argentina. Studies on Neotropical Fauna and Environment 40:1-5.

Glade, A. 1988. Libro Rojo de los Vertebrados Terrestres de Chile. Corporación Nacional Forestal, Santiago, Chile.

Hofmann, R.R. 1989. Evolutionary steps of ecophysiological adaptation and diversification of ruminants: A comparative view of their digestive system. Oecologia 78: 443-457.

Holechek, J.L. \& M. Vavra 1981. The effect of slide and frequency observation numbers on the precision of microhistological analysis. Journal of Range Management 34:337-338. 
Holechek, J.L. \& B.D. Gross 1982. Evaluation of different calculation procedures for microhistological analysis. Journal of Range Management 36:721-723.

Jiménez, J., O. Guineo, P. Corti, J.A. Smith, W. Flueck, A. Vila, Z. Gizejewski, R. Gill, B. McShea \& V. Geist 2008. Hippocamelus bisulcus. En 2008 IUCN Red List of Threatened Species. http://www.iucnredlist.org

Krebs, C. 1989. Ecological Methodology. Harper and Row Publisher, New York, USA.

Latour, M. \& A. Pelliza Sbriller 1981. Clave para la determinación de la dieta de herbívoros en el noroeste de la Patagonia. Revista Investigación Agrícola (INTA) 16:109-157.

López, R., A. Serret, R. Faúndez \& G. Palé 1998. Estado del conocimiento actual de la distribución del huemul (Hippocamelus bisulcus, Cervidae) en Argentina y Chile. FVSA, WWF \& CODEFF, Concepción, Chile.

López, R., R. Figueroa, E. S. Corales, A. Trostel \& S. Alvarado 2001. Estudio del huemul en los Nevados de Chillán - Laguna de la Laja. CODEFF y Sociedad Zoológica de Frankfurt, Concepción, Chile.

Merino, M. 1995. Dieta del huemul (Hippocamelus bisulcus) en el Canal Moyano - Glaciar Viedma, P.N. Los Glaciares, Pcia. de Santa Cruz, Argentina. Boletín Técnico FVSA 25: 18-25.

Quintana, R.D, N. Madanes, P. Vuillermoz \& P. Otero 2000. Relevamiento ambiental del Campo "Los Huemules", Provincia de Santa Cruz: "Caracterización ecológica y evaluación de su condición como unidad de manejo y conservación". Informe del Programa de RVS de la FVSA, Buenos Aires, Argentina.

Redford, K. \& J. Eisenberg. 1992. Mammals of the Neotropics (Vol. 2): The Southern Cone. The University of Chicago Press, Chicago, USA.

Sepúlveda, L., A. Pelliza \& M. Manacorda 2004. Importancia de los tejidos no epidérmicos en el microanálisis de dieta. Ecología Austral 14:31-38.
Serret, A. 1992. Distribución actual del huemul (Hippocamelus bisculus) en la República Argentina. Boletín Técnico № 1 . Fundación Vida Silvestre Argentina, Buenos Aires.

Serret, A. 2001. El huemul: Fantasma de la Patagonia. Zagier \& Urruty Publicaciones, Buenos Aires, Argentina.

Sierralta, D. 2001. Faecal microhistology, study of the diet of huemul. En Saucedo, C. \& A. Echenique (eds) Huemul Ecology Research for Conservation Planning: Final Conference, Darwin Project. CONAF, RI, FRA \& MLURI, Coyhaique, Chile.

Sparks, D. \& J.C. Malechek 1968. Estimating percentage dry weight in diets using a microscopic technique. Journal of Range Management 21:264-265.

Vila, A.R., R. López, H. Pastore, R. Faúndez \& A. Serret 2006. Current distribution and conservation of the huemul (Hippocamelus bisulcus) in Argentina and Chile. Mastozoología Neotropical 13: 263-269.

Vila A.R., G. Galende \& H. Pastore 2009a. Feeding ecology of the endangered huemul (Hippocamelus bisulcus) in Los Alerces National Park, Argentina. Mastozoología Neotropical 16: 423-431.

Vila, A.R., Borrelli, L. \& L. Martínez 2009b. Dietary overlap between huemul and livestock in Los Alerces National Park. Journal of Range Management 73, 368-373.

Vila, A.R., C.E. Saucedo, D. Aldridge, E. Ramilo \& P. Corti 2010. South Andean Huemul (Hippocamelus bisulcus, Molina 1782). Pp: 89-100, En Barbanti Duarte, J.M. \& S. González (eds.) Neotropical Cervidology: Biology and Medicine of Latin American Deer. FUNEPIUCN, San Pablo, Brasil.

Williams, O.B. 1969. An improved technique for identification of plant fragments in herbivore feces. Journal of Range Management 22: 51-52. 
A. R. VILA et al. 